\title{
Advanced intermediate reflector layers for thin film silicon tandem solar cells
}

\author{
Franz-Josef Haug, Mathieu Boccard, Remi Biron, Björn Niesen, Matthieu Despeisse, Christophe Ballif
}

Ecole Polytechnique Fédérale de Lausanne (EPFL), Institute of Microengineering, Photovoltaics and Thin Film Electronics Laboratory, Rue A.-L. Breguet 2, CH-2000 Neuchâtel, Switzerland

\begin{abstract}
Tandem solar cells based on thin film silicon benefit from an intermediate reflector layer between the top and bottom cells since it enhances the absorption in the top cell. The top cell can thus be manufactured thinner and less prone to light induced degradation. Made from a thin layer of nanocrystalline silicon oxide, the interlayer provides a second functionality since it aids in the spatial separation of local shunts occurring in both sub-cells. Recently, the reflector morphology received attention since it can provide a third function; here, a substantial difference exists between the commonly used configurations, i.e. superstrate or substrate. In the former, the thin layer of nanocrystalline silicon oxide reproduces the morphology of the underlying top cell. Its surface may thus be too rough for the growth of the bottom cell. In the latter configuration, reflectors made from a thick layer of $\mathrm{ZnO}$ can yield an adequate texture for the top cell, but conductive $\mathrm{ZnO}$ loses the effect of shunt quenching. We present our recent progress with improved intermediate reflector layers in both cell types. For silicon oxide based interlayers, we introduce a smoothing lacquer layer with self-organized openings that allow current transport. For $\mathrm{ZnO}$ based interlayers, we demonstrate that a treatment in oxygen plasma is capable of tuning the in-plane resistivity.
\end{abstract}

Index Terms - amorphous silicon, nanocrystalline silicon, tandem cells

\section{INTRODUCTION}

Thin film silicon remains to be an attractive material for solar cells, but the high performance of other thin film technologies puts it under increasing pressure. Demonstrating high efficiency is therefore crucial. Thin film silicon has the unique advantage that amorphous and nanocrystalline material make an ideal pair for advanced tandem cells. After the first demonstration of micromorph tandems by J. Meier [1], it was soon noted that current matching would require unsuitably thick top cells. This issue was successfully overcome by the introduction of an intermediate reflector layer (IRL) made from a low index material like $\mathrm{ZnO}$ [2]. The IRL concept received renewed attention after $\mathrm{K}$. Yamamoto successfully replaced ex-situ $\mathrm{ZnO}$ by in-situ $\mathrm{SiOx}$ doped with phosphorous [3] and was recently also demonstrated in triple junction solar cells [4]. In tandem cells, the top cell current was observed to increase with increasing IRL thickness up to a maximum value found between 90 and $120 \mathrm{~nm}$ [5]; for larger thickness, an oscillatory behavior indicates interference effects whose amplitude depend on the roughness of the underlying texture [6]. With increasing oxygen content, the in-plane conductivity rapidly drops but IRLs with values as low as $10^{-10} 1 / \Omega \mathrm{cm}$ have been reported to maintain high $\mathrm{FF}$ in tandem solar cells [7, 8]. In a microstructural analysis, Cuony found that $\mathrm{SiOx}$ grown with very high hydrogen dilution consists of a phase separated material where thin conductive filaments of silicon are embedded into a matrix of silicon oxide [9]. The unique microstructure thus yields sufficient out-of-plane conductivity for the series connection of the sub-cells. At the same time, moderate lateral transport in the $\mathrm{SiOx}$ film isolates shunts that occur on strongly textured morphologies.

Parallel to these developments applying to the superstrate configuration on glass, tandem cells in the n-i-p configuration on flexible substrates proceeded along a different direction. Since their process flow starts with the deposition of the nanocrystalline bottom cell, it is mandatory to adapt the light scattering texture of the substrate to this sub-cell. Then, the growth of the bottom cell usually results in a smoother morphology that is little suited for light scattering into the top cell. In this environment, a SiOx-based IRL yields only a moderate enhancement of the top cell current by interference [10]. A much more substantial enhancement was demonstrated with a thick layer of $\mathrm{ZnO}$ grown by low pressure chemical vapor deposition (LPCVD) [11].

In this contribution, we discuss the challenges that are related to standard IRL solutions. For both configurations, we propose novel approaches to manufacture advanced multifunctional IRLs that enable top cell current boost together with morphology adaptation.

\section{EXPERIMENTAL}

All experiments presented in this contribution were performed on $\mathrm{ZnO}$ films grown by LPCVD. After growth, the surface textures consisting of randomly oriented pyramids were exposed to a surface treatment in Ar-plasma. Thicknesses of $2.5 \mu \mathrm{m}$ and $5 \mu \mathrm{m}$ with treatments lasting for 4 ' and $45^{\prime}$ were used for superstrate and substrate cells, respectively. These electrodes are denoted by Z5/2-4 and Z545 , respectively. The silicon films are grown by PECVD as published elsewhere $[12,13]$.

For the intermediate reflector layers in superstrate cells, the following structure is used: the top cell finishes with a $90 \mathrm{~nm}$ thick n-type SiOx which forms the first part of the IRL, followed by a $10 \mathrm{~nm}$ thick $\mathrm{ZnO}$ layer that serves as etch-stop. Then, a UV-curable lacquer is spin coated, dried on a hotplate, cured in UV-light, and subjected to reactive etching in 
$\mathrm{SF}_{6}-\mathrm{O}_{2}$ plasma. More details on the process are described elsewhere [14]. In the reference cells, a $140 \mathrm{~nm}$ thick SiOx intermediate reflector (SOIR) [5] is used (50 $\mathrm{nm}$ in addition to the $90 \mathrm{~nm}$ n-layer).

In substrate cells, a $1.1 \mu \mathrm{m}$ thick LPCVD-ZnO film is used as IRL. Since this film provides its typical pyramidal texture quite independent of the underlying morphology, it is sometimes called asymmetric intermediate reflector (AIR).

After IRL deposition, the second sub-cell is grown to form a tandem device and the cell areas are defined. After depositing a $2.5 \mu \mathrm{m}$ thick $\mathrm{ZnO}$ film (Z5/2) to form the second electrode, the area between the cells is cleared by lift-off and reactive ion etching of the silicon films. Finally, measurements of the $\mathrm{j}-\mathrm{V}$ characteristics are performed under a dual-source sunsimulator (WACOM) and the current-densities in the sub-cells are determined by measuring the external quantum-efficiency.

\section{RESULTS AND DISCUSSION}

\section{A. Smoothing IRLs for superstrate solar cells}
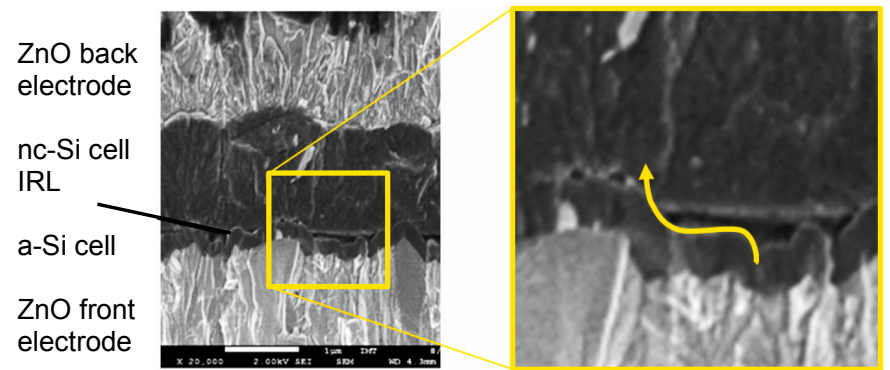

Fig. 1: Cross section through a superstrate tandem solar cell on a 2.5 $\mu \mathrm{m}$ thick front electrode, the growth sequence from bottom to top. The enlargement illustrates the smooth growth template provided by the lacquer film. The arrow indicates the current path around the insulating lacquer layer towards a point of contact.

The smoothing effect of the novel IRL is shown in Fig. 1. Since the lacquer fills preferentially the valleys of the underlying structure, short etching exposes the the tips of the morphology and the $\mathrm{SiOx}: \mathrm{P} / \mathrm{ZnO}$ bilayer can thus establish a conductive path to the nanocrystalline cell.

Deep trenches of the underlying morphology are still covered with the lacquer layer whose surface resembles water floating around higher ground. In the solar cell parameters shown in Fig. 2, the most striking gain concerns the open circuit voltage (Voc) where the cells with smoothening IRL gain $40 \mathrm{mV}$ with respect to the reference. Among the two different etching conditions, higher Voc is obtained with the shorter 2' etching which is expected to expose less of the rough morphology, thus nucleating fewer zones of defective material in the nanocrystalline bottom cell.

The FF values shown in Fig. 2 indicate that the presence of insulating regions does not harm the device performance. The best value is again found for the shorter treatment, even though the strong current mismatch of all cells in this experiment prevents a clear conclusion. Similar results, but for cells with higher bottom-cell currents and thus better matching are discussed in [14].

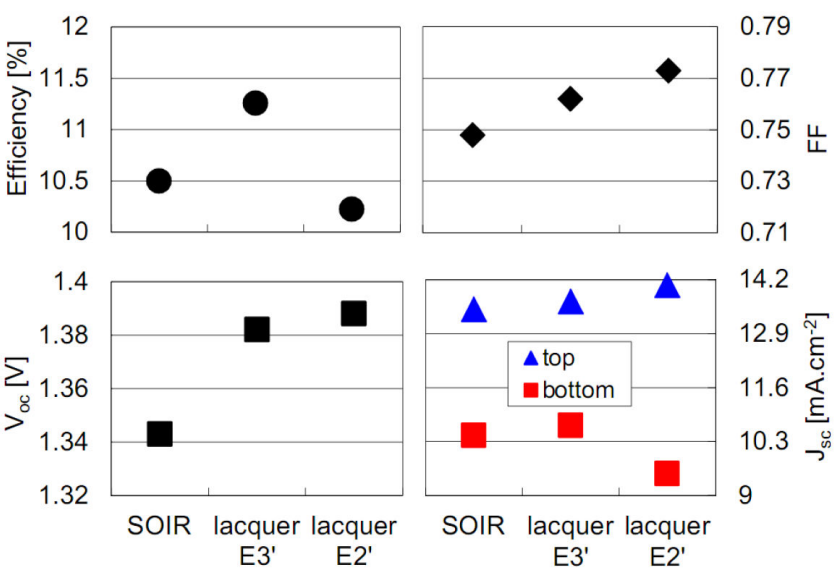

Fig. 2: Solar cell parameters of superstrate tandem cells with smoothing IRLs. SOIR denotes a $140 \mathrm{~nm}$ thick SiOx:P layer, E3 and E2 refer to lacquer layers etched for 3 and 2 minutes, respectively.

\section{B. ZnO IRLs for substrate solar cells}

The effect of a thick $\mathrm{ZnO}$ based IRL for substrate solar cells is shown in Fig. 3. Note that this cell is deposited on a Z5-45 substrate which provides large features for light scattering in the nanocrystalline bottom cell. Owing to the long plasma treatment, sharp features are well rounded off. This bottom cell did not show any zones of defective material, resulting in an open circuit voltage of $510 \mathrm{mV}$ in a single junction reference device of $1.7 \mu \mathrm{m}$ thickness [13].

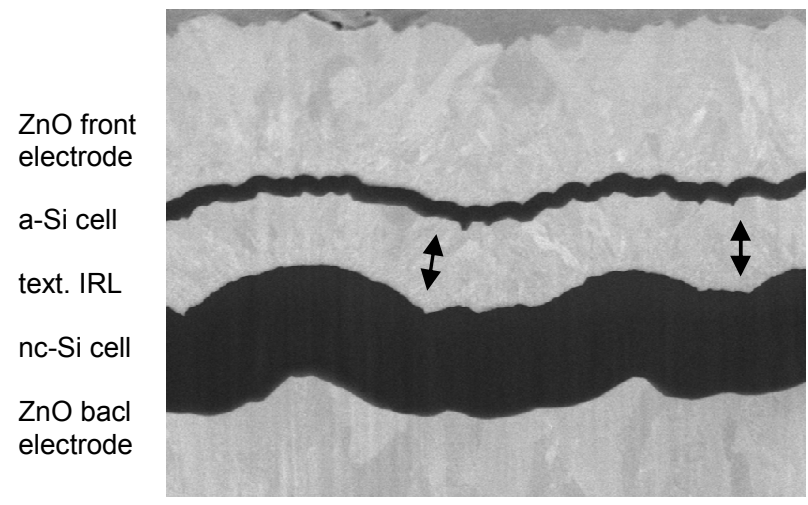

Fig. 3: Cross section through a substrate solar cell on $5 \mu \mathrm{m}$ thick $\mathrm{ZnO}$ treated for $45^{\prime}$. The arrows show how pinched areas become covered by the thick $\mathrm{ZnO}$ intermediate layer.

The arrows in Fig. 3 indicate areas prone to pinching, i.e. where the absorber layer might start to develop zones of bad material quality if it were grown any thicker. Such areas can also compromise the growth and performance of the top cell, except that the thick ZnO IRL covers them very effectively by means of its different growth dynamics, establishing thus an adequate growth template for the top cell. 


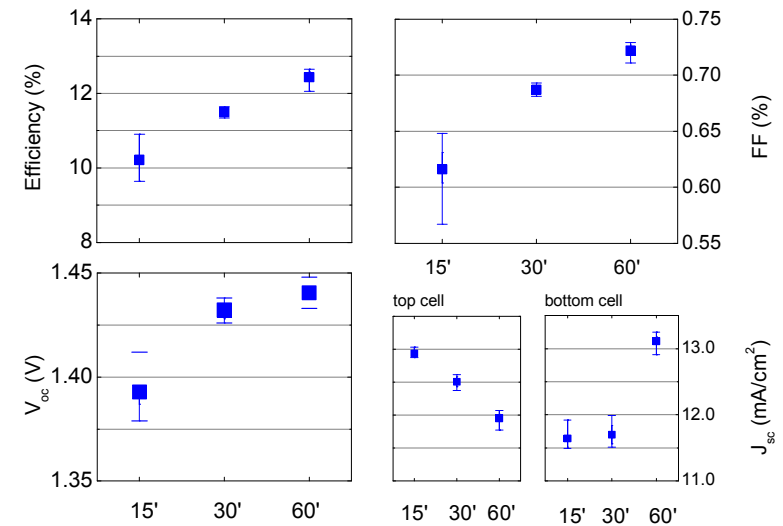

Fig. 4: Solar cell parameters of substrate solar cells with thick $\mathrm{ZnO}$ IRLs treated for 15,30 , and 60 minutes, changing thus the IRL conductivity from conductive over intermediate to semi-insulating and the IRL roughness from high over intermediate to small.

Different from the plasma treatment in argon which is applied to the $\mathrm{ZnO}$ of the back electrode, the aim of treating the interlayer is mainly to increase its resistivity. Nevertheless, the oxygen plasma also yields morphological changes. Owing to is lower atomic mass, oxygen treatments lasting for 15,30 and 60 minutes reduce the initial rms roughness of $70 \mathrm{~nm}$ to 60,50 , and $35 \mathrm{~nm}$, respectively, as measured on reference layers on glass [15]. The associated reduction in light scattering is visible in the reduction of the top cell current density shown in Fig. 4.

The open circuit voltage and the FF improve, suggesting a beneficial influence of the oxygen treatment. At this point it is not clear whether resistivity or morphological effects dominate. Such a conclusion will require a similar series with Ar-plasma which does not change the resistivity.

\section{CONCLUSIONS}

We presented two examples of advanced intermediate reflector layers in thin film silicon tandem cells. Applying a self-masking technique on the existing texture, we created an open network of low index material that provides reflection by a high index contrast to the surrounding silicon films as well as sufficient electric contact to interconnect the two sub-cells of the tandem cell. In a second example, we demonstrated that a plasma treatment in oxygen can be used to tune the resistivity of the intermediate reflector layer, thus suppressing the lateral connection of shunted regions and their associated current drains. Both examples show that the processing is compatible with state-of-the-art efficiencies.

\section{ACKNOWLEDGEMENT}

We thankfully acknowledge funding by the Velux Foundation, by the European Union within the projects Silicon-Light (No. 241277) and FastTrack (No. 283501), and by the Swiss Federal Office of Energy (No. SI/500750-01).

\section{REFERENCES}

[1] J. Meier, et al. "Intrinsic microcrystalline silicon - a promising new thin film solar cell material", in Proc. 1st World PVSEC Hawaii, p. 409-412, (1994)

[2] D. Fischer, et al. "The micromorph solar cell: extending a a-Si:H technology towards thin film crystalline silicon", in Proc. 25th IEEE PVSC Washington D. C., p. 1053, (1996)

[3] K. Yamamoto, et al. "High efficiency thin film silicon hybrid cell and module with newly develeoped innovative interlayer", in Proc. 4th World PVSEC Barcelona, p. 1489, (2006)

[4] B. Yan, et al. "Effect of dual-function nano-structured silicon oxide thin film on multi-junction solar cells", in Proc. 37th IEEE PVSC Seattle, p. 002560, (2011)

[5] P. Buehlmann, et al., "In situ silicon oxide based intermediate reflector for thin-film silicon micromorph solar cells", Applied Physics Letters 91(14), p. 143505 (2007).

[6] D. Dominé, et al., "Optical management in high-efficiency thin-film silicon micromorph solar cells with a silicon oxide based intermediate reflector", Physica Status Solidi - Rapid Research Letters 2(4) (2008).

[7] A. Lambertz, T. Grundler, and F. Finger, "Hydrogenated amorphous silicon oxide containing a microcrystalline silicon phase and usage as an intermediate reflector in thinfilm silicon solar cells", Journal of Applied Physics 109(11), p. 113109-113109-10 (2011).

[8] T. Grundler, A. Lambertz, and F. Finger, "N-type hydrogenated amorphous silicon oxide containing a microcrystalline silicon phase as an intermediate reflector in silicon thin film solar cells", physica status solidi (c) 7(3-4), p. 1085-1088 (2010).

[9] P. Cuony, et al., "Silicon filaments in silicon oxide for next-generation photovoltaics", Advanced Materials 24(9), p. 1182 (2012).

[10] F.-J. Haug, et al., "Development of micromorph tandem solar cells on flexible low cost plastic substrates", Solar Energy Materials and Solar Cells 93, p. 884-8877 (2009).

[11] T. Söderström, et al., "Asymmetric intermediate reflector for tandem micromorph thin film silicon solar cells", Applied Physics Letters 94, p. 063501 (2009).

[12] M. Boccard, et al., "Nanometer- and micrometer-scale texturing for high-efficiency micromorph thin-film silicon solar cells", IEEE Journal of Photovoltaics 2(2), p. 83-87 (2012).

[13] R. Biron, et al., "Optimization of the asymmetric intermediate reflector morphology for high stabilized efficiency thin n-i-p micromorph solar cells", IEEE Journal of Photovoltaics 3(1), p. 41-45 (2013).

[14] M. Boccard, et al., "Smoothing intermediate reflecting layer for tandem thin-film silicon solar cells", Availabe online, Solar Energy Materials and Solar Cells (2013).

[15] R. Biron, "Layers with advanced optoelectronic properties for high-efficiency micromrph solar cells on opaque substrates", PhD thesis, EPFL, (2013) 